\title{
Single top quark at future hadron colliders: Complete signal and background study
}

\author{
A. S. Belyaev \\ Instituto de Física Teórica, Universidade Estadual Paulista, Rua Pamplona 145, 01405-900 São Paulo, Brazil \\ and Skobeltsyn Institute of Nuclear Physics, Moscow State University 119899 Moscow, Russian Federation \\ E. E. Boos \\ Skobeltsyn Institute of Nuclear Physics, Moscow State University 119899 Moscow, Russian Federation \\ L. V. Dudko \\ Skobeltsyn Institute of Nuclear Physics, Moscow State University 119899 Moscow, Russian Federation
}

(Received 10 June 1998; published 16 February 1999)

\begin{abstract}
We perform a detailed theoretical study including decays and jet fragmentation of all the important modes of single top quark production and all the basic background processes at the upgraded Fermilab Tevatron and CERN LHC colliders. Special attention is paid to the complete tree level calculation of the QCD fake background which was not considered in previous studies. An analysis of the various kinematical distributions for the signal and backgrounds allow us to work out a set of cuts for an efficient background suppression and extraction of the signal. It is shown that the signal to background ratio after optimized cuts could reach about 0.4 at the Tevatron and 1 at the LHC. The remaining after cuts signal rate at the LHC for the lepton+jets signature is expected to be about $6.1 \mathrm{pb}$ and will be enough to study single top quark physics even during LHC operation at a low luminosity. [S0556-2821(99)00605-0]

PACS number(s): $12.60 . \mathrm{Cn}$
\end{abstract}

\section{INTRODUCTION}

The existence of the top quark was established in 1995 by the Collider Detector at Fermilab (CDF) and D Collaborations at the Tevatron collider [1]. The top quark has been discovered in the strong $t \bar{t}$ pair production mode.

The cross section of the electroweak process of the single top quark was found to be comparable with QCD top quark pair production [2]. The single top quark production mechanism is an independent way of confirming the existence of the top quark and a straightforward key to measuring the $V t b$ Cabibbo-Kobayashi-Maskawa (CKM) matrix element and studying the $W t b$ vertex. Since the mass of the top quark is very large compared to all other quarks, one might expect some deviations from standard model (SM) predictions of top quark interactions [3]. The single top quark production rate is directly proportional to the $W t b$ coupling and therefore it is a promising place to look for deviations from the SM.

However, one should stress that the task of background reduction is a much more serious and important problem in the case of the single top quark compared to $t \bar{t}$-pair production. It happens because the jet multiplicity of single top quark events is typically less than for $t \bar{t}$-pair production and so QCD $W j j$ and multijet backgrounds are much higher, and the problem of the single top quark signal extraction is more involved. That is why a detailed background study is especially needed in order to find an optimal strategy to search for the single top quark.

The top quark decays into a $W$ boson and a $b$ quark with an almost $100 \%$ branching ratio in the framework of the standard model. We consider here the subsequent leptonic decays of the $W$ boson to an electron (muon) and neutrino, as this signal has much less background and should be easier to find experimentally than channels with hadronic decay of the $W$ boson.

\section{MC SIMULATION}

In order to study the possibility of signal extraction from the background we have created a Monte Carlo (MC) generator for a complete set of single top quark production and background processes. The generator was designed as a new external user process for the PYTHIA 5.7/JETSET 7.4 package [4]. This generator is related to PYTHIA 5.7 by a special interface and uses FORTRAN codes of squared matrix elements produced by the package COMPHEP [5]. For integration over phase space and the consequent event simulation the Monte Carlo generator uses kinematics with a proper smoothing of singular variables [6] from COMPHEP and the integrator package BASES/SPRING [7].

The effects of final state radiation, hadronization, and string jet fragmentation (by means of JETSET 7.4) have also been taken into account. The following resolutions have been used for the jet and electron energy smearing: $\Delta E^{\text {had }} / E$ $=0.5 / \sqrt{E}$ and $\Delta E^{e l e} / E=0.2 / \sqrt{E}$. In our analysis we used the cone algorithm for jet reconstruction with cone size $\Delta R$ $=\sqrt{\Delta \varphi^{2}+\Delta \eta^{2}}=0.5$. The minimum $E_{T}$ threshold for a cell to be considered as a jet initiator has been chosen as $5 \mathrm{GeV}$ while the one of the summed $E_{T}$ for a collection of cells to be accepted as a jet has been chosen as $10 \mathrm{GeV}$.

For all calculations the CTEQ3M parton distribution has been used. For top quark production we chose the QCD $Q^{2}$ scale equal to the top quark mass squared, while for the $W j j$ background $Q^{2}=M_{W}^{2}$ has been taken. For calculations of $j b \bar{b}$ and $j j b \bar{b}$ processes we chose the invariant $b \bar{b}$ mass for the $Q^{2}$ scale. 

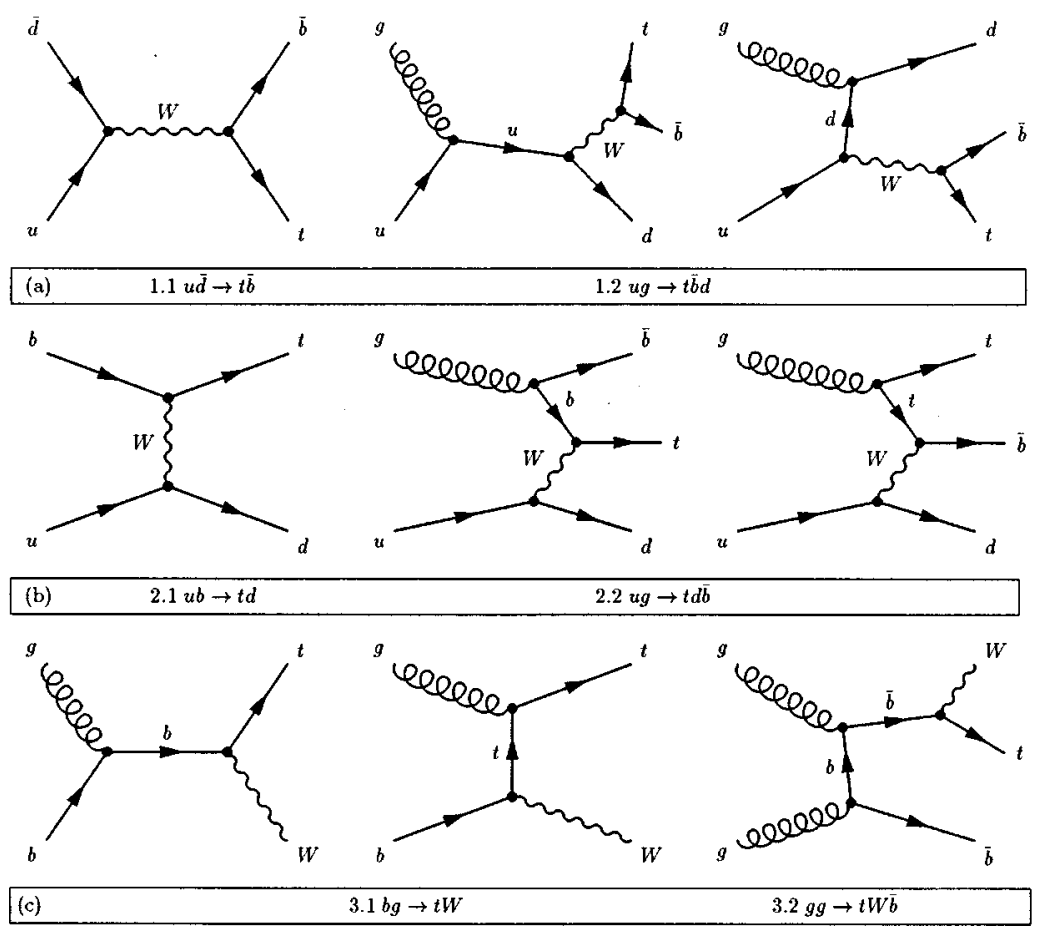

FIG. 1. Diagrams for single top quark production.
Under the assumptions mentioned above the kinematic features of both signatures for signal and background have been studied.

\section{A. Signal}

We concentrated on the following set of processes at the Tevatron $p \bar{p}$ and LHC $p p$ colliders leading to single top quark production: (1) $p \bar{p} \rightarrow t q \bar{b}+X$, (2) $p \bar{p} \rightarrow t \bar{b}+X$, (3) $p \bar{p} \rightarrow t q+X$, (4) $p \bar{p} \rightarrow t W+X$, where $q$ is a light quark and $X$ represents the remnants of the proton and antiproton. Basic Feynman diagrams for the processes mentioned above are shown in Fig. 1. We refer to Ref. [8], which considers the whole set of Feynman diagrams for signal subprocesses.

It is necessary to stress that the $p \bar{p} \rightarrow t W+X$ contributes only $5 \%$ to the total cross section at the $2 \mathrm{TeV}$ upgraded Tevatron. It could be easily omitted at Tevatron energies but should be taken into account for CERN Large Hadron Collider (LHC) energies since as we shall demonstrate below its contribution at the LHC will be about $30 \%$ of the the total cross section of single top quark production.

For event analysis we have rescaled the total cross sections of single top quark production using the results of the next-to-leading (NLO) calculations from the papers in Ref. [9] $\left(m_{t}=175 \mathrm{GeV}\right)$ : for $\sqrt{s}=2 \mathrm{TeV}, \sigma(t \bar{b})=0.88$ $\pm 0.05 \mathrm{pb}, \quad \sigma(W g=t q b \bar{b}+t q)=2.43 \pm 0.4 \mathrm{pb}, \quad$ and for $\sqrt{s}=14 \mathrm{TeV}, \quad \sigma(t \bar{b})=10.2 \pm 0.6 \mathrm{pb}, \quad \sigma(W g=t q b \bar{b}+t q)$ $=245 \pm 9 \mathrm{pb}$.

We found the LO cross section of $p \bar{p} \rightarrow t W+X$ equal to $98 \mathrm{pb}$ at the LHC with about $8 \%$ uncertainty due to the choice of different structure functions. The cross section for $p \bar{p} \rightarrow t W^{-}+X$ was calculated in the following way. The process $g g \rightarrow t W \bar{b}$ has been combined (diagram 3.2 in Fig. 1c shows the only one among eight different topologies contributed to this subprocesses) with $b g \rightarrow t W$ (diagram 3.1 in Fig. 1c). Similar to the $W$-gluon fusion process the subtraction of the gluon splitting term has been made in order to avoid a double counting:

$$
\begin{aligned}
\sigma(g b+g g \rightarrow t W+X)_{r e a l}= & \sigma(g b \rightarrow t W)+\sigma(g g \rightarrow t W \bar{b}) \\
& -\sigma(g \rightarrow b \bar{b} \otimes g b \rightarrow t W)
\end{aligned}
$$

In previous studies the two above subprocess $g g \rightarrow t W \bar{b}$ [10] and $g b \rightarrow t W$ [11] have been considered separately, which being simply added leads to an overestimation and stronger scale dependence of the cross section. In addition we calculated the complete set of Feynman diagrams for $u \bar{u}+d \bar{d} \rightarrow t W \bar{b}$ subprocesses.

However, one should also take into account that the complete set of Feynman diagrams for Wtb processes includes the $t \bar{t}$-pair production subprocess. The $t \bar{t}$ pair production is one of the background processes included separately in the analysis. Therefore its contribution has been removed from the $t W+X$ rate. It can be done in a gauge invariant way by excluding the kinematical region of the $W b$ invariant mass around the top quark mass within three top quark decay widths as was used in case of $e^{+} e^{-}$collisions [12]:

$$
M_{t}+3 \Gamma_{t o p}>M_{W b}>M_{t}+3 \Gamma_{t o p} .
$$

For our calculations we used $M_{t}=175 \mathrm{GeV}, \Gamma_{\text {top }}$ $=1.59 \mathrm{GeV}$.

As a result, we have $91 \mathrm{pb}$ for the $\sigma(g b+g g \rightarrow t W$ $+X)_{\text {real }}$ process, while $\sigma(u \bar{u}+d \bar{d} \rightarrow t W \bar{b}+X)$ process gives additional $7 \mathrm{pb}$ to the total $p \bar{p} \rightarrow t W^{-}+X$ cross section which is therefore expected to be about $98 \mathrm{pb}$ at the LHC. 

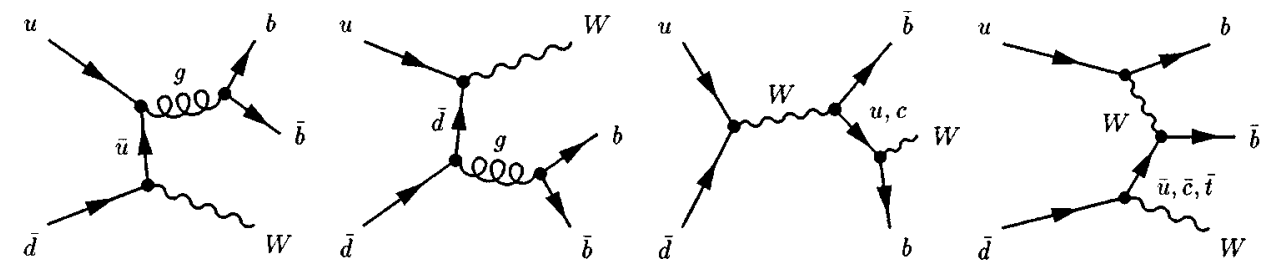

(a)

(b)
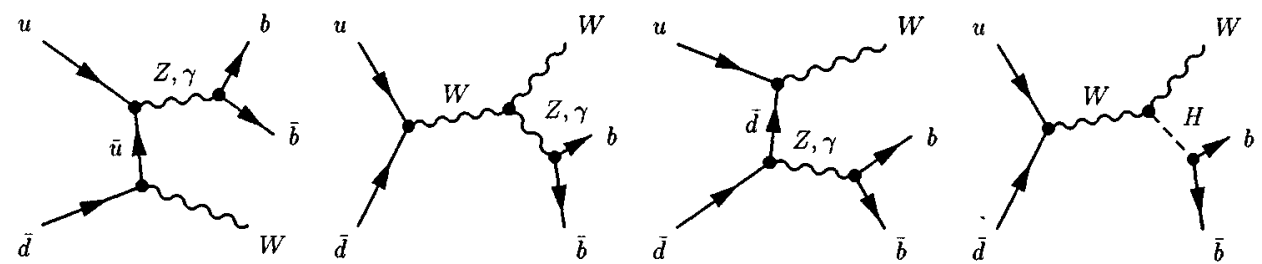

FIG. 2. Diagrams for $W b \bar{b}$ background.

(c)

(d)

Thus the total single top quark rate is expected to be about $3.5 \mathrm{pb}$ at the upgraded Tevatron and $350 \mathrm{pb}$ at the LHC to be compared to the $t \bar{t}$ production rates [13] $7.6 \mathrm{pb}$ and $760 \mathrm{pb}$, respectively. The relative contributions of different subprocesses to the total single top quark production cross section at Tevatron are the following: $p \bar{p} \rightarrow t q \bar{b}(39 \%), p \bar{p} \rightarrow t b$ $(30 \%), p \bar{p} \rightarrow t q(26 \%), p \bar{p} \rightarrow t W+X(5 \%)$.

The relative contributions of $p \bar{p} \rightarrow t b$ and $p \bar{p} \rightarrow t W+X$ turn over at LHC: $p \bar{p} \rightarrow t q \bar{b}(44.2 \%), p \bar{p} \rightarrow t b \quad(2.8 \%), p \bar{p}$ $\rightarrow t q(25 \%), p \bar{p} \rightarrow t W+X(28 \%)$.

As was mentioned we consider here the leptonic decay modes of $W$ bosons from the top quark and therefore the final state signature to search for the signal will be $e^{ \pm}\left(\mu^{ \pm}\right)+\boldsymbol{E}_{T}$ +2 (3) jets where one of the jets is the $b$-quark jet from the top quark decay.

\section{B. Main backgrounds}

The main backgrounds leading to the same $e^{ \pm}\left(\mu^{ \pm}\right)$ $+\boldsymbol{E}_{T}+2$ (3) jets final state signature as the single top quark signal are the following: $p \bar{p} \rightarrow W+2$ (3) jets (gluonic, $\alpha \alpha_{s}$ order, and electroweak $\alpha^{2}$ order), $p \bar{p} \rightarrow t \bar{t}$ pair top quark production, and $j(j) b \bar{b}$ QCD fake background when one jet imitates the electron.

All numbers for $W j j(W b \bar{b})$ and $j(j) b \bar{b}$ backgrounds are, for the initial general cuts on jets, $\Delta R_{j j(e j)}>0.5, p_{t}$ jet $>10$ $\mathrm{GeV}$ for Tevatron and $\Delta R_{j j(e j)}>0.5, p_{t}$ jet $>20 \mathrm{GeV}$ for LHC.

The total cross section of $\mathrm{W}+2$ jets "gluonic" background is more than two orders of magnitude higher than the signal one. This process includes 32 subprocesses for $u, d$ quarks and gluons in the initial state [14], and the total cross section equals $1240 \mathrm{pb}$ for Tevatron and $7500 \mathrm{pb}$ for LHC ( $s$ and $c$ sea quarks give additional $3 \%$ contribution to the total cross section).

The specific feature of single top quark production is the high energetic $b$ jet in the final state and one additional $b$ jet for $W$-gluon and $W^{*}$ processes. It is clear that the only chance to extract the signal from such an overwhelming background is the efficient $b$-quark identification. We assume $50 \%$ of a double $b$-tagging efficiency hereafter.

However, the cross section of the $W+2$ jet process is so large that even with the requirement of a double $b$ tagging but due to a $b$-quark jet misidentification it represents an important part of the total background. In our study we chose $0.5 \%$ misidentification probability which is based on the previous MC analysis [15].

The $b$-quark content of the $W+2$ jet processes is fairly small - less then $1 \%$. For the cuts mentioned above the total cross section for the $W^{ \pm} b \bar{b}$ process (gluon splitting) is 8.7 $\mathrm{pb}$ for the Tevatron and $30 \mathrm{pb}$ for the LHC. However, the $W+2 b-$ jet process is the irreducible part of the total background which has different kinematical properties from the main QCD $W+2$ jet part, and as will be shown below it depends differently on the cuts.

Therefore the $W^{ \pm} b \bar{b}$ background has been considered separately and we have calculated it completely.

The complete set of Feynman background diagrams for the $W b \bar{b}$ final state is shown in Fig. 2 for $u$ and $\bar{d}$ quarks in the initial state. The main contribution comes from subprocess (a) with the gluon splitting into the $b \bar{b}$ quark pair. Diagrams with virtual photons (c) contribute only $1 \%$ to the total cross section. The contribution from $W Z$ process (c) can be suppressed by applying a cut on the invariant $b \bar{b}$ mass. The LO cross section for $W Z$ is 2.5 pb at the Tevatron and about $30 \mathrm{pb}$ at the LHC. For our analysis we apply $K$ factor $=1.33$ (1.55) for the Tevatron (LHC) to rescale this number for the NLO total cross section [16]. The cross section of the process (d) of Higgs boson production (we take as an example $m_{H}=110 \mathrm{GeV}$ ) is even smaller: $0.16(1.8) \mathrm{pb}(\mathrm{LO})$ at the Tevatron (LHC), and it means the Higgs boson is really not an important background for the single top quark. We apply $K$ factor $=1.25$ (1.1) for the Tevatron (LHC) to rescale the results for the NLO one [17]. Diagrams (b) give a very small contribution due to small value of CKM elements.

An important background is top quark pair production: when one of the top quarks decays hadronically and another one leptonically. One of the cut which helps to reduce the 
background is the cut on the number of jets which was required to be less than 4. At the parton level this cut reduces the top quark pair rate very strongly. However, at the simulation level with a hadronization and jet reconstruction being taken into account the reduction of this background is not so strong anymore. And as a result the top quark pair represents an important part of the background. This fact will be shown below. The NLO total cross section for $t t$-pair production was taken [13] at the Tevatron to be equal to $7.56 \mathrm{pb}$ and 760 $\mathrm{pb}$ at the LHC.

Another kind of important reducible background comes from multijet QCD processes. This happens due to a possible misidentification of the jet as an electron in the detector. Though the probability of that is very small (of order of $0.01-0.03 \%$ ) [18], the cross section for such processes is huge and gives a significant contribution to the background for single top quark production. For the analysis we took $\epsilon_{\text {fake }}=0.02 \%$.

We have calculated the total cross section and made MC simulations for $j b b$ and $j j b b$ processes which are relevant for our signature if one of the light jets imitates an electron. In such a way we could simulate the basic distributions of the expected fake background and understand how strong it could be suppressed by kinematical cuts. The MC simulation of the fake background for the single top quark study is presented for the first time.

The background from the light jets appears to be less important despite the fact that the light jet cross sections even with cuts are very large. The light jet background is suppressed by three small factors, by the double mistag probability of identifying a light jet as a $b$ jet and by the small fake probability of identifying a light jet as an electron. For instance, the $g g \rightarrow g g g$ subprocess could contribute to the background when two gluon jets fake $b$-quark jets and a third gluon jet fakes the lepton. The cross section of $g g \rightarrow g g g$ itself is huge but as was mentioned its contribution to the single top quark background is suppressed by the fake probability of a gluon multiplied by the double mistag probability of two gluons. For example, the cross section of triple gluon production at the Tevatron is $2.7 \times 10^{7} \mathrm{pb}$, the double mistag gluon probability is equal to $10^{-6}$, and the fake probability of a gluon is of order $10^{-4}$. Therefore the contribution from the $g g \rightarrow g g g$ process is estimated to be equal to $\simeq 10^{-2} \mathrm{pb}$ (we use combinatoric factor 3 here) and one can neglect it.

In Table I and Table II all subprocesses giving $j b b$ and $j j b b$ final state signatures are shown respectively with the corresponding cross sections for the Tevatron and LHC. In our calculations we neglected the double sea quark and $c$ sea quark small contributions. The total cross section for $j b b$ at the Tevatron (LHC) is 240 and 70 (511 and 362) nb for $j b b$ and $j j b b$.

The cross section of the $j b b$ process in Table I is only about 2 times higher at the LHC than at the Tevatron because higher jet $p_{T}$ cuts for the LHC have been used $(20 \mathrm{GeV}$ at the LHC and $10 \mathrm{GeV}$ at the Tevatron). If equal jet $p_{T}$ cuts are used, the cross section at the LHC is about 50 times higher than that at the Tevatron.
TABLE I. Total cross section for $j b \bar{b}$ process for the Tevatron and LHC. The following cuts have been applied at the parton level calculations: $\Delta R_{j j}>0.5, p_{t}$ jet $>10 \mathrm{GeV}$ for the Tevatron and $\Delta R_{j j(e j)}>0.5, p_{t}$ jet $>20 \mathrm{GeV}$ for the LHC.

\begin{tabular}{lcc}
\hline \hline Process & Tevatron $(\mathrm{pb})$ & LHC $(\mathrm{pb})$ \\
\hline$g g \rightarrow g b \bar{b}$ & $1.64 \times 10^{5}$ & $3.91 \times 10^{5}$ \\
$g \bar{u}(\bar{u} g) \rightarrow \bar{u} b \bar{b}$ & $1.80 \times 10^{4}\left(2.50 \times 10^{3}\right)$ & $5.61 \times 10^{3}\left(5.61 \times 10^{3}\right)$ \\
$g u(u g) \rightarrow u b \bar{b}$ & $2.50 \times 10^{4}\left(1.80 \times 10^{4}\right)$ & $2.41 \times 10^{4}\left(2.41 \times 10^{4}\right)$ \\
$g \bar{d}(\bar{d} g) \rightarrow \bar{d} b \bar{b}$ & $9.00 \times 10^{3}\left(3.21 \times 10^{3}\right)$ & $6.61 \times 10^{3}\left(6.61 \times 10^{3}\right)$ \\
$g \bar{s}(\bar{s} g) \rightarrow \bar{s} b \bar{b}$ & $1.97 \times 10^{3}\left(1.97 \times 10^{3}\right)$ & $4.49 \times 10^{3}\left(4.49 \times 10^{3}\right)$ \\
$g d(d g) \rightarrow d b \bar{b}$ & $3.21 \times 10^{3}\left(9.00 \times 10^{3}\right)$ & $1.38 \times 10^{4}\left(1.38 \times 10^{4}\right)$ \\
$g s(s g) \rightarrow s b \bar{b}$ & $1.97 \times 10^{3}\left(1.97 \times 10^{3}\right)$ & $4.49 \times 10^{3}\left(4.49 \times 10^{3}\right)$ \\
$d \bar{d}(\bar{d} d) \rightarrow g b \bar{b}$ & $5.67 \times 10^{2}\left(1.25 \times 10^{2}\right)$ & $2 . .82 \times 10^{2}\left(2.82 \times 10^{2}\right)$ \\
$u \bar{u}(\bar{u} u) \rightarrow g b \bar{b}$ & $1.31 \times 10^{3}\left(8.61 \times 10^{1}\right)$ & $4.16 \times 10^{2}\left(4.16 \times 10^{2}\right)$ \\
Total & $2.40 \times 10^{5} \mathrm{pb}$ & $5.11 \times 10^{5} \mathrm{pb}$ \\
\hline \hline
\end{tabular}

We performed two ways of calculating the $j j b \bar{b}$ process: the complete tree level calculation and the splitting approximation when one uses the complete result from $j b \bar{b}$ with an additional jet radiation from initial and final states. In such a way we have checked the validity of the splitting approximation.

As was expected the splitting approximation works reasonably well for the total rate if rather soft cuts on the additional jet are used and the difference increases if the stronger cuts are applied. Table III illustrates such a difference in results for the approximation and exact calculations for various cuts on the $p_{T}$ of the second jet (that is, the light jet with the smallest $p_{T}$ which is more likely an additional radiated light jet).

Indeed one can see that for the $p_{j 2 T}>10 \mathrm{GeV}$ cut the difference between the exact calculation and the splitting approximation is only about $10 \%: 70 \mathrm{nb}$ and $64 \mathrm{nb}$, respectively. But after the $p_{j 2 T}>40 \mathrm{GeV}$ cut those results differ almost by a factor of 5: one has 1.2 and $0.25 \mathrm{nb}$ for the exact calculation of $j j b \bar{b}$ and splitting approximation, respectively.

The expected difference in the distribution on the momenta transverse of the second jet is illustrated in Fig. 3. The distribution in the case of the splitting approximation is significantly softer.

Since we do not apply a high $p_{T}$ cut on the jet (one of them fakes electrons, for which we apply a $15 \mathrm{GeV} p_{T}$ cut), the difference between the exact calculation and the approximation is of the order of $25 \%$ for the fake background simulation.

\section{Signal and background kinematical properties}

The rate of the signal and backgrounds presented above clearly shows that even after $b$ tagging the signal is still more than one order less than the background. This fact requires a special kinematical analysis in order to find out a strategy of how to suppress the background and extract the signal in an optimal way. 
TABLE II. Total cross section for $j j b \bar{b}$ process for the Tevatron and LHC. The following cuts have been applied at the parton level calculations: $\Delta R_{j j}>0.5, p_{t}$ jet>10 GeV for the Tevatron and $\Delta R_{j j(e j)}$ $>0.5, p_{t}$ jet $>20 \mathrm{GeV}$ for the LHC.

\begin{tabular}{|c|c|c|}
\hline Process & Tevatron $(\mathrm{pb})$ & LHC (pb) \\
\hline$u u \rightarrow u u b \bar{b}$ & $1.23 \times 10^{2}$ & $1.17 \times 10^{3}$ \\
\hline$u \bar{u}(\bar{u} u) \rightarrow b \bar{b} b \bar{b}$ & $2.55 \times 10^{0}-$ & $1.06 \times 10^{0}\left(1.06 \times 10^{0}\right)$ \\
\hline$u \bar{u}(\bar{u} u) \rightarrow s \bar{s} b \bar{b}$ & $6.61 \times 10^{0}-$ & $2.53 \times 10^{0}\left(2.53 \times 10^{0}\right)$ \\
\hline$u \bar{u}(\bar{u} u) \rightarrow c \bar{c} b \bar{b}$ & $6.52 \times 10^{0}-$ & $2.53 \times 10^{0}\left(2.53 \times 10^{0}\right)$ \\
\hline$u \bar{u}(\bar{u} u) \rightarrow d \bar{d} b \bar{b}$ & $6.66 \times 10^{0}-$ & $2.52 \times 10^{0}\left(2.53 \times 10^{0}\right)$ \\
\hline$u \bar{u}(\bar{u} u) \rightarrow u \bar{u} b \bar{b}$ & $8.70 \times 10^{2}-$ & $3.38 \times 10^{2}\left(3.38 \times 10^{2}\right)$ \\
\hline$u \bar{u}(\bar{u} u) \rightarrow g g b \bar{b}$ & $2.15 \times 10^{2}-$ & $8.92 \times 10^{1}\left(8.92 \times 10^{1}\right)$ \\
\hline$u d(d u) \rightarrow u d b \bar{b}$ & $1.44 \times 10^{2}\left(4.20 \times 10^{1}\right)$ & $7.40 \times 10^{2}\left(7.40 \times 10^{2}\right)$ \\
\hline$u s(s u) \rightarrow u s b \bar{b}$ & $9.63 \times 10^{1} \longrightarrow$ & $1.71 \times 10^{2}\left(1.71 \times 10^{2}\right)$ \\
\hline$u \bar{d}(\bar{d} u) \rightarrow u \bar{d} b \bar{b}$ & $3.73 \times 10^{2}\left(1.20 \times 10^{2}\right)$ & $3.74 \times 10^{2}\left(3.74 \times 10^{2}\right)$ \\
\hline$u \bar{s}(\bar{s} u) \rightarrow u \bar{s} b \bar{b}$ & $9.63 \times 10^{1} \longrightarrow$ & $1.71 \times 10^{2}\left(1.71 \times 10^{2}\right)$ \\
\hline$d \bar{u}(\bar{u} d) \rightarrow d \bar{u} b \bar{b}$ & $3.73 \times 10^{2}\left(1.20 \times 10^{2}\right)$ & $1.78 \times 10^{2}\left(1.78 \times 10^{2}\right)$ \\
\hline$s \bar{u}(\bar{u} s) \rightarrow \bar{u} s b \bar{b}$ & $9.63 \times 10^{1} \longrightarrow$ & $\longrightarrow$ \\
\hline $\bar{u} \bar{u} \rightarrow \bar{u} \bar{u} b \bar{b}$ & $9.10 \times 10^{1} \longrightarrow$ & - \\
\hline $\bar{u} \bar{d}(\bar{d} \bar{u}) \rightarrow \bar{u} \bar{d} b \bar{b}$ & $4.20 \times 10^{1}\left(1.44 \times 10^{2}\right)$ & - \\
\hline $\bar{u} \bar{s}(\bar{s} \bar{u}) \rightarrow \bar{u} \bar{s} b \bar{b}$ & $-\left(9.63 \times 10^{1}\right)$ & $\longrightarrow-$ \\
\hline$d d \rightarrow d d b \bar{b}$ & $6.40 \times 10^{1}$ & $1.17 \times 10^{3}$ \\
\hline$d \bar{d}(\bar{d} d) \rightarrow b \bar{b} b \bar{b}$ & $9.38 \times 10^{-1}\left(9.38 \times 10^{-1}\right)$ & $7.00 \times 10^{-1}\left(7.00 \times 10^{-1}\right)$ \\
\hline$d \bar{d}(\bar{d} d) \rightarrow s \bar{s} b \bar{b}$ & $2.40 \times 10^{0}\left(2.40 \times 10^{0}\right)$ & $1.70 \times 10^{0}\left(1.70 \times 10^{0}\right)$ \\
\hline$d \bar{d}(\bar{d} d) \rightarrow c \bar{c} b \bar{b}$ & $2.35 \times 10^{0}\left(2.35 \times 10^{0}\right)$ & $1.70 \times 10^{0}\left(1.70 \times 10^{0}\right)$ \\
\hline$d \bar{d}(\bar{d} d) \rightarrow d \bar{d} b \bar{b}$ & $2.24 \times 10^{2}\left(2.24 \times 10^{2}\right)$ & $2.05 \times 10^{2}\left(2.05 \times 10^{2}\right)$ \\
\hline$d \bar{d}(\bar{d} d) \rightarrow u \bar{u} b \bar{b}$ & $2.40 \times 10^{0}\left(2.40 \times 10^{0}\right)$ & $1.70 \times 10^{0}\left(1.70 \times 10^{0}\right)$ \\
\hline$d \bar{d}(\bar{d} d) \rightarrow g g b \bar{b}$ & $7.30 \times 10^{1}\left(7.30 \times 10^{1}\right)$ & $5.86 \times 10^{1}\left(5.86 \times 10^{1}\right)$ \\
\hline $\bar{d} \bar{d} \rightarrow \bar{d} \bar{d} b \bar{b}$ & $5.10 \times 10^{1}$ & - \\
\hline$d \bar{s}(\bar{s} d) \rightarrow d \bar{s} b \bar{b}$ & $4.23 \times 10^{1}-$ & $9.16 \times 10^{1}\left(9.16 \times 10^{1}\right)$ \\
\hline$d s(s d) \rightarrow d s b \bar{b}$ & $4.23 \times 10^{1}-$ & $9.16 \times 10^{1}\left(9.16 \times 10^{1}\right)$ \\
\hline $\bar{d} s(s \bar{d}) \rightarrow \bar{d} s b \bar{b}$ & $-4.23 \times 10^{1}$ & $9.16 \times 10^{1}\left(9.16 \times 10^{1}\right)$ \\
\hline $\bar{d} \bar{s}(\bar{s} \bar{d}) \rightarrow \bar{d} \bar{s} b \bar{b}$ & $-4.23 \times 10^{1}$ & $9.16 \times 10^{1}\left(9.16 \times 10^{1}\right)$ \\
\hline$g u(u g) \rightarrow g u b \bar{b}$ & $7.28 \times 10^{2}\left(7.82 \times 10^{3}\right)$ & $2.37 \times 10^{4}\left(2.37 \times 10^{4}\right)$ \\
\hline$g \bar{u}(\bar{u} g) \rightarrow g \bar{u} b \bar{b}$ & $7.82 \times 10^{3}\left(7.28 \times 10^{2}\right)$ & $4.53 \times 10^{3}\left(4.53 \times 10^{3}\right)$ \\
\hline$g d(d g) \rightarrow g d b \bar{b}$ & $1.01 \times 10^{3}\left(3.52 \times 10^{3}\right)$ & $1.29 \times 10^{4}\left(1.29 \times 10^{4}\right)$ \\
\hline$g s(s g) \rightarrow g s b \bar{b}$ & $7.61 \times 10^{2}\left(7.61 \times 10^{2}\right)$ & $2.43 \times 10^{3}\left(2.43 \times 10^{3}\right)$ \\
\hline$g \bar{d}(\bar{d} g) \rightarrow g \bar{d} b \bar{b}$ & $3.52 \times 10^{3}\left(1.01 \times 10^{3}\right)$ & $5.47 \times 10^{3}\left(5.47 \times 10^{3}\right)$ \\
\hline$g \bar{s}(\bar{s} g) \rightarrow g \bar{s} b \bar{b}$ & $7.61 \times 10^{2}\left(7.61 \times 10^{2}\right)$ & $2.43 \times 10^{3}\left(2.43 \times 10^{3}\right)$ \\
\hline$g g \rightarrow b \bar{b} b \bar{b}$ & $9.90 \times 10^{1}$ & $6.58 \times 10^{2}$ \\
\hline$g g \rightarrow s \bar{s} b \bar{b}$ & $4.80 \times 10^{2}$ & $2.21 \times 10^{3}$ \\
\hline$g g \rightarrow c \bar{c} b \bar{b}$ & $4.60 \times 10^{2}$ & $2.24 \times 10^{3}$ \\
\hline$g g \rightarrow d \bar{d} b \bar{b}$ & $3.85 \times 10^{2}$ & $2.11 \times 10^{3}$ \\
\hline$g g \rightarrow u \bar{u} b \bar{b}$ & $3.85 \times 10^{2}$ & $2.11 \times 10^{3}$ \\
\hline$g g \rightarrow g \bar{g} b \bar{b}$ & $3.62 \times 10^{4}$ & $2.43 \times 10^{5}$ \\
\hline Total & $7.01 \times 10^{4} \mathrm{pb}$ & $3.62 \times 10^{5} \mathrm{pb}$ \\
\hline
\end{tabular}

The distributions for several sensitive kinematical variables for a separation of the signal and the background are shown in Fig. 4 for the Tevatron and in Fig. 5 for the LHC.
The above-mentioned effects of the jet fragmentation, detector resolution, and energy smearing are included in the figures. Among the kinematical variables for the separation of 
TABLE III. Comparison the cross sections for $j j b \bar{b}$ process for exact calculations and the splitting approximation for various $p_{j 2 T}$ cuts at Tevatron. The following cuts have been applied at the MC level: $\Delta R_{j j}>0.5, p_{t}$ of the first jet $>10 \mathrm{GeV}$.

\begin{tabular}{lcccc}
\hline \hline$p_{j 2 T}[\mathrm{GeV}]$ & 10 & 15 & 20 & 40 \\
\hline$\sigma_{j j b b}^{\text {exact }}[\mathrm{pb}]$ & 70 & 32 & 14 & 1.2 \\
$\sigma_{j j b b}^{\text {split }}[\mathrm{pb}]$ & 64 & 22 & 8 & 0.25 \\
\hline \hline
\end{tabular}

the signal and background the most attractive were found to be the following.

(i) $p_{T}$ of leading jet: $p_{T}$ of the leading jet distribution for the signal has a peak around $m_{\text {top }} / 3$, while it is much softer for the QCD background at the Tevatron (Fig. 4). The main difference between kinematical distributions for signal and background at the Tevatron is that jets from $W+j j$ and $j(j) b b$ processes are softer and less central than those for signals with one very hard jet coming from the top quark and another softer jet, accompanying the top quark. For the LHC there is no such striking difference in $p_{T}$ of the leading jet distribution between signal and background. It happens because of a higher c.m. energy and the dominating contribution to the background from $t \bar{t}$ production (Fig. 5).

(ii) $\sqrt{\hat{s}}$-invariant mass of the system (Figs. 4,5 ): it is always bigger for signal than those for $W j j$ and $j(j) b b$ backgrounds. It is important to notice that the $t \bar{t}$ background peaks at higher values of the invariant mass of the system which is clearly seen in the case of the LHC, where this background dominates.

(iii) $p_{T} W$ : the $W$ boson tends to be harder from top quark decay than from QCD processes.

(iv) Scalar transverse energy $H_{T}$ (Fig. 4c,5c), $H_{T}$ $=\mid E_{T}($ jet 1$)|+| E_{T}\left(\right.$ jet2) $|+| E_{T}$ (lepton) $\mid:$ this kinematical

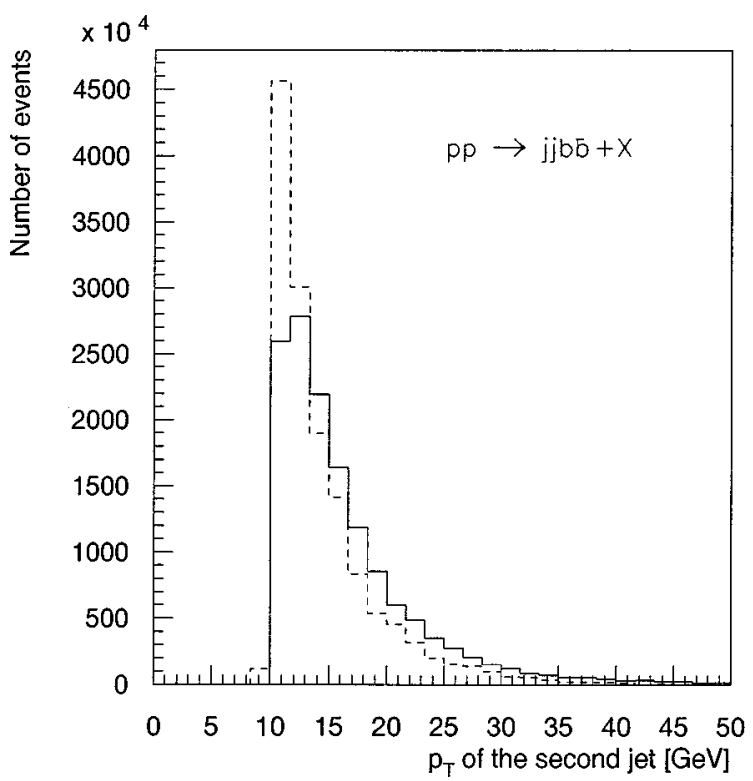

FIG. 3. Distribution for $p_{T}$ of the second jet for the $j j b \bar{b}$ process for the exact calculation (solid line) and for splitting from the $j b \bar{b}$ process (dashed line) at the Tevatron.
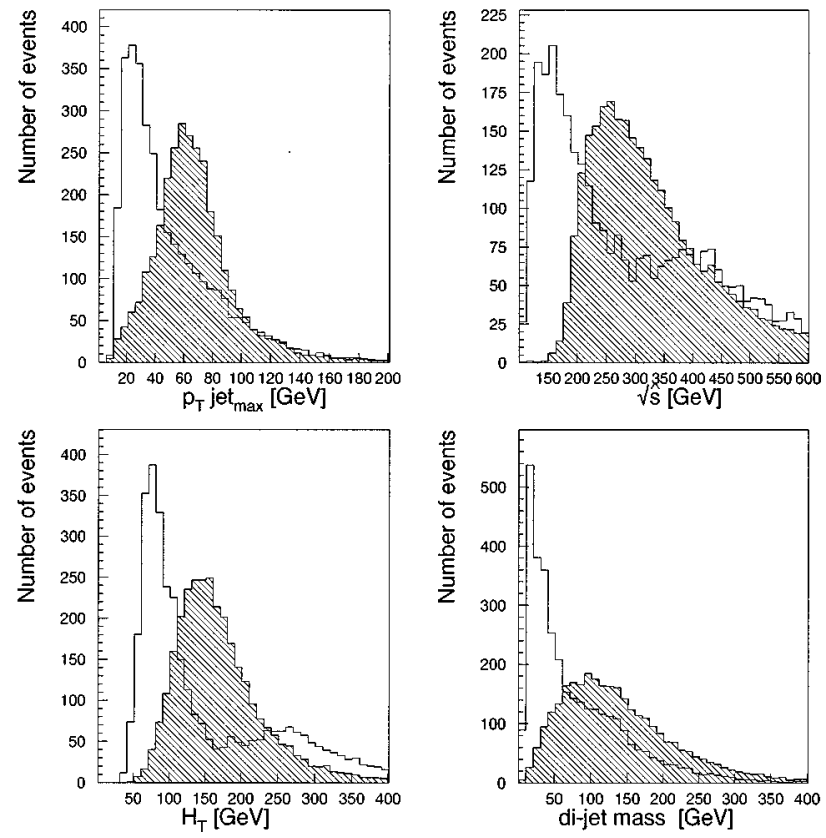

FIG. 4. Distributions for signal and background for the some most spectacular variables at the Tevatron. The sketched histogram stands for signal.

variable has a peak around $150 \mathrm{GeV}$ for the signal, around $300 \mathrm{GeV}$ for the $t \bar{t}$ background, and peaks at small values for the QCD background.

(v) dijet mass (Figs. 4,5): It is harder for the signal than for the QCD background, for which the $b \bar{b}$ pair coming mainly from gluon splitting, in the same time dijet mass distribution of $t \bar{t}$ background, has a similar shape as the signal. The dijet mass cut is also used for a reduction of the $W Z$ background.
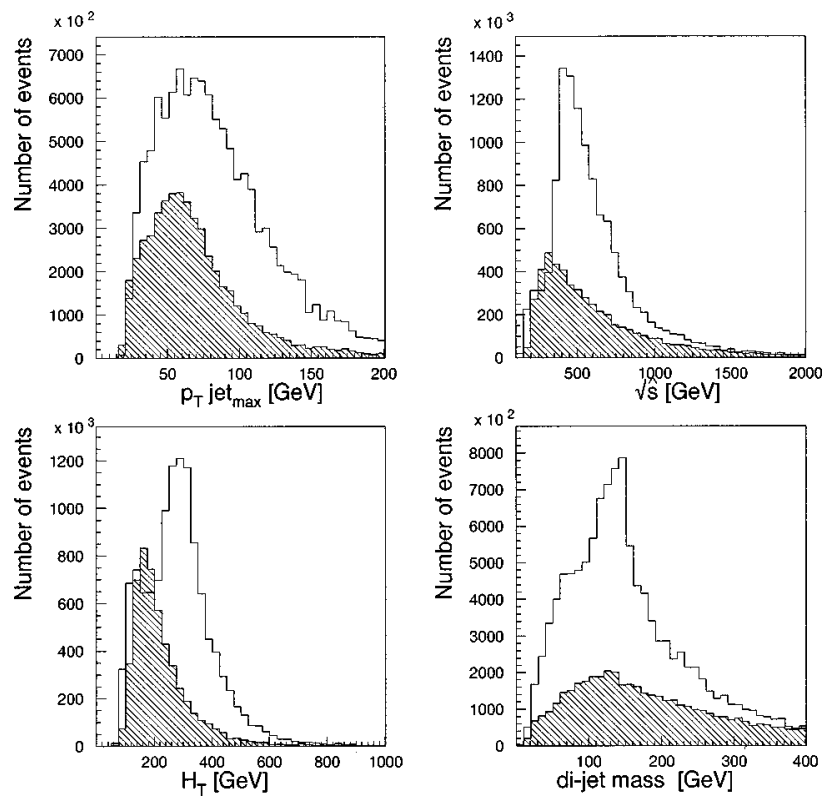

FIG. 5. Distributions for signal and background for the some most spectacular variables at the LHC. The sketched histogram stands for signal. 
TABLE IV. Number of events for the single top quark signal and background at the Tevatron. The cuts numbering corresponds to (four) sets of cuts with their consequent application. The window $\pm 50 \mathrm{GeV}$ around the $175 \mathrm{GeV}$ bin was imposed for a reconstructed "effective" top quark mass.

\begin{tabular}{lccccccc}
\hline \hline Cuts & Signal & $W b \bar{b}$ & $W j j$ & $W Z$ & $j(j) b \bar{b}$ & $t \bar{t}$ & $W H$ \\
\hline Cut 1 & $1.986 \times 10^{2}$ & $3.680 \times 10^{2}$ & $2.644 \times 10^{2}$ & $2.059 \times 10^{1}$ & $6.292 \times 10^{2}$ & $5.849 \times 10^{2}$ & $8.428 \times 10^{0}$ \\
Cut 2 & $1.514 \times 10^{2}$ & $1.711 \times 10^{2}$ & $1.034 \times 10^{2}$ & $1.136 \times 10^{1}$ & $1.114 \times 10^{2}$ & $4.898 \times 10^{2}$ & $6.491 \times 10^{0}$ \\
Cut 3 & $1.493 \times 10^{2}$ & $1.453 \times 10^{2}$ & $9.211 \times 10^{1}$ & $1.053 \times 10^{1}$ & $1.030 \times 10^{2}$ & $4.898 \times 10^{2}$ & $6.278 \times 10^{0}$ \\
Cut 4 & $1.295 \times 10^{2}$ & $1.173 \times 10^{2}$ & $7.687 \times 10^{1}$ & $8.564 \times 10^{0}$ & $8.910 \times 10^{1}$ & $4.191 \times 10^{2}$ & $5.145 \times 10^{0}$ \\
Cut 5 & $1.286 \times 10^{2}$ & $1.107 \times 10^{2}$ & $7.488 \times 10^{1}$ & $8.515 \times 10^{0}$ & $8.353 \times 10^{1}$ & $4.186 \times 10^{2}$ & $5.124 \times 10^{0}$ \\
Cut 6 & $1.249 \times 10^{2}$ & $1.038 \times 10^{2}$ & $6.649 \times 10^{1}$ & $8.087 \times 10^{0}$ & $6.961 \times 10^{1}$ & $4.185 \times 10^{2}$ & $5.013 \times 10^{0}$ \\
Cut 7 & $1.247 \times 10^{2}$ & $1.031 \times 10^{2}$ & $6.649 \times 10^{1}$ & $7.419 \times 10^{0}$ & $4.455 \times 10^{1}$ & $1.055 \times 10^{2}$ & $4.562 \times 10^{0}$ \\
Cut 8 & $1.216 \times 10^{2}$ & $8.867 \times 10^{1}$ & $6.141 \times 10^{1}$ & $7.266 \times 10^{0}$ & $3.619 \times 10^{1}$ & $1.039 \times 10^{2}$ & $4.490 \times 10^{0}$ \\
\hline
\end{tabular}

Signal 122, background 297; $\mathrm{S} / \mathrm{B} \simeq 0.41$

In our analysis we used an "effective" invariant top quark mass variable which is constructed using the following algorithm. It is clear that mass of the top quark decaying to a lepton, neutrino, and $b$ quark cannot be unambiguously reconstructed the since the $z$ component of the neutrino cannot be measured. One can construct the top quark mass

$$
m_{t}^{2}=\left(P_{e}+P_{\nu}+P_{b}\right)^{2}
$$

using one of two solutions for $p_{z \nu}$ of the simple quadratic equation $^{1}$

$$
m_{W}^{2}=\left(P_{e}+P_{\nu}\right)^{2}=80.12^{2} .
$$

Our Monte Carlo analysis shows that if one chooses $p_{z \nu}$ to be the $\left|p_{z \nu}\right|_{\text {min }}$ from two solutions, then it will be in about $70 \%$ true $p_{z \nu}$. In fact the reason for that is obvious and related to the fact that smaller values of $p_{z \nu}$ correspond in most cases to smaller values of the total invariant mass $\sqrt{s}$. And for smaller values of $\sqrt{s}$ the effective parton-parton luminosity is larger and therefore the cross section is higher. However, anyway, if the one solution for $p_{z \nu}$ is used, the invariant "effective" mass distribution is broader than the real invariant top quark mass and one should apply a rather wide window for this kinematical variable in order not to lose too many signal events. We chose a $\pm 50 \mathrm{GeV}$ window in our analysis.

Based on such different behaviors of the signal and background kinematical distributions the following set of cuts for the background suppression has been worked out.

$$
\begin{gathered}
\text { Cut 1: } \Delta R_{j j(e j)}>0.5, \quad p_{T} \text { jet }>10 \mathrm{GeV}, \\
\boldsymbol{E}_{T}>15 \mathrm{GeV}, \quad p_{t e}>15 \mathrm{GeV}
\end{gathered}
$$

for the Tevatron and

$$
\Delta R_{j j(e j)}>0.5, \quad p_{t} \text { jet }>20 \mathrm{GeV},
$$

\footnotetext{
${ }^{1}$ Such a method of single top quark mass reconstruction is known and has been used in the past [see Ref. [19]].
}

$$
\boldsymbol{E}_{T}>20 \mathrm{GeV}, \quad p_{t e}>20 \mathrm{GeV}
$$

for the LHC which are "initial" cuts for jet separation and $W$-boson identification.

Cut 2: $p_{t}$ jet $_{\max }>45 \mathrm{GeV}$.

Cut 3: $\sqrt{\hat{s}}>180 \mathrm{GeV}$.

Cut 4: $p_{T} W>30 \mathrm{GeV}$.

Cut 5: dijet mass $>25 \mathrm{GeV}$.

Cut 6: $H_{T}>100 \mathrm{GeV}$ for Tevatron and $260 \mathrm{GeV}>H_{T}$

$>100 \mathrm{GeV}$ for $\mathrm{LHC}$.

Cut $7: 3 \geqslant n$ jet $\geqslant 2$.

Cut 8: dijet mass $\geqslant 40 \mathrm{GeV}$.

The effect of the consequent application of this set of cuts is presented in Tables IV and V. We should stress that the "effective" mass window cut $\pm 50 \mathrm{GeV}$ was an initial cut and has been applied along with all other cuts shown in the tables.

The number of events presented in the tables as well as in Figs. 4 and 5 corresponds to the total integrated luminosity $2 \mathrm{fb}^{-1}\left(100 \mathrm{fb}^{-1}\right),{ }^{2}$ for the Tevatron (LHC), under the above-mentioned assumptions of a double $b$-tagging efficiency of $50 \%$ and a $b$-quark mistagging probability of $0.5 \%$.

From the tables one can see that in fact two cuts, cut 2 reducing the QCD $+W j j$ background and cut 7 eliminating the $t \bar{t}$ background, play the leading role. At the same time all cuts are strongly correlated and one can effectively replace cut 2 by cut $3+4$ or a more complicated combination with the same success.

\footnotetext{
${ }^{2}$ The numbers for the LHC could be easily rescaled to the $30 \mathrm{fb}^{-1}$ of the low luminosity LHC operation.
} 
TABLE V. Number of events for the single top quark signal and background at the LHC. The cuts numbering corresponds to (four) set of cuts with their consequent application. The window $\pm 50 \mathrm{GeV}$ around the $175 \mathrm{GeV}$ bin was imposed for a reconstructed "effective" top quark mass.

\begin{tabular}{lccccccc}
\hline \hline Cuts & Signal & $W b \bar{b}$ & $W j j$ & $W Z$ & $j(j) b \bar{b}$ & $t \bar{t}$ & $W H$ \\
\hline Cut 1 & $1.212 \times 10^{6}$ & $8.236 \times 10^{4}$ & $1.724 \times 10^{5}$ & $1.912 \times 10^{4}$ & $1.155 \times 10^{6}$ & $4.449 \times 10^{6}$ & $6.124 \times 10^{3}$ \\
Cut 2 & $8.792 \times 10^{5}$ & $5.143 \times 10^{4}$ & $1.058 \times 10^{5}$ & $1.177 \times 10^{4}$ & $6.112 \times 10^{5}$ & $3.762 \times 10^{6}$ & $4.923 \times 10^{3}$ \\
Cut 3 & $8.764 \times 10^{5}$ & $.871 \times 10^{4}$ & $1.015 \times 10^{5}$ & $1.138 \times 10^{4}$ & $6.053 \times 10^{5}$ & $3.762 \times 10^{6}$ & $4.854 \times 10^{3}$ \\
Cut 4 & $7.423 \times 10^{5}$ & $3.826 \times 10^{4}$ & $7.758 \times 10^{4}$ & $9.048 \times 10^{3}$ & $4.974 \times 10^{5}$ & $3.262 \times 10^{6}$ & $3.976 \times 10^{3}$ \\
Cut 5 & $7.401 \times 10^{5}$ & $3.771 \times 10^{4}$ & $7.735 \times 10^{4}$ & $9.013 \times 10^{3}$ & $4.957 \times 10^{5}$ & $3.262 \times 10^{6}$ & $3.972 \times 10^{3}$ \\
Cut 6 & $5.643 \times 10^{5}$ & $3.649 \times 10^{4}$ & $7.524 \times 10^{4}$ & $7.545 \times 10^{3}$ & $4.729 \times 10^{5}$ & $6.214 \times 10^{5}$ & $3.334 \times 10^{3}$ \\
Cut 7 & $5.370 \times 10^{5}$ & $3.610 \times 10^{4}$ & $7.408 \times 10^{4}$ & $6.122 \times 10^{3}$ & $2.411 \times 10^{5}$ & $1.886 \times 10^{5}$ & $2.740 \times 10^{3}$ \\
Cut 8 & $5.296 \times 10^{5}$ & $3.177 \times 10^{4}$ & $7.019 \times 10^{4}$ & $6.030 \times 10^{3}$ & $2.301 \times 10^{5}$ & $1.886 \times 10^{5}$ & $2.694 \times 10^{3}$ \\
\hline
\end{tabular}

Signal $5.3 \times 10^{5}$, background $5.3 \times 10^{5} ; \mathrm{S} / \mathrm{B}=1.0$

The strong background reduction is clearly illustrated in Figs. $6 a, b$ and $7 \mathrm{a}, \mathrm{b}$ for the invariant top quark mass distribution before (a) and after (b) application of kinematical cuts. After the cuts are applied the background became about 10 times smaller at the Tevatron and 18 times at the LHC while approximately $60 \%(40 \%)$ of the signal survived at the Tevatron (LHC). The signal/background ratio becomes equal approximately to 0.4 at the Tevatron and 1 at the LHC. Such a background suppression will allow one to measure the signal cross section with high accuracy.

The cross section for single top quarks includes the Wtb coupling directly, in contrast to $t \bar{t}$ pair production. Therefore, single top quark production provides a unique opportunity to study the $W t b$ structure and to measure $V_{t b}$. Experimental studies of this type are among the main goals of single top quark physics. Using the single top quark search one can examine the effects of a deviation in the Wtb coupling from the SM structure and directly measure the CKM matrix element $V_{t b}$. Since the signal to background ratio is high after kinematical cuts are applied, the error of $V_{t b}$ measurement as was shown in [8] is expected to be of the order of $10 \%$ at the Tevatron run 2 . In the same time much higher statistics and good signal/background ratio at the LHC considerably improve the measurement of the $V_{t b}$ value and test the $W t b$ vertex. Since the statistical error for $10^{5}$ events is less than $1 \%$, then the uncertainty of the $W t b$ vertex mea-
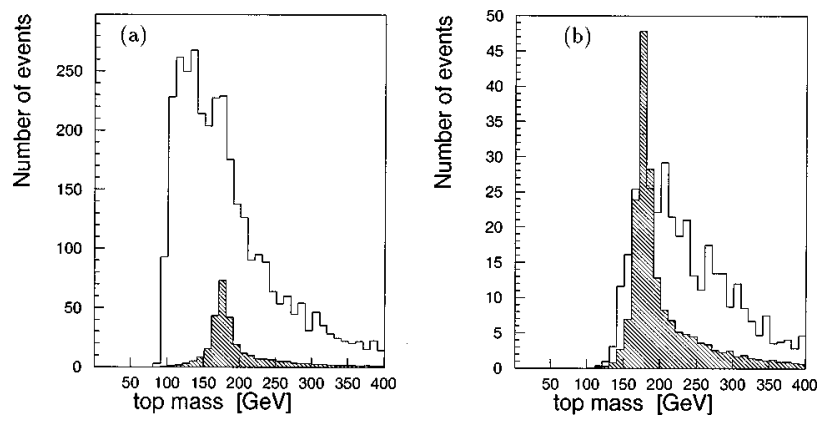

FIG. 6. Distributions for invariant top quark mass before (a) and after (b) cut application at the Tevatron. The sketched histogram stands for signal. surement at the LHC depends mostly on the uncertainty of theoretical calculations for the single top quark production cross section and for the backgrounds. That is why calculations of the next order corrections to single top quark production including corrections to the kinematical distributions but not only to the total event rate and a simulation of the main backgrounds at the NLO level are important for the LHC.

Another important source of uncertainties in the Wtb vertex measurement comes from the parton distribution uncertainty as well as from the accuracy of top quark measurements. In the case of the Tevatron these uncertainties have been taken into consideration [8]. However, in the case of the LHC this point is not very clear since one does not know how large those uncertainties would remain when the experiment will start, and the parton distribution functions and the top quark mass will be measured in separate experiments. That is why at the present stage we did not include the pointed uncertainties for the case of the LHC.

\section{CONCLUSIONS}

The study of single top quark production versus complete background processes has been done. For calculations a special generator has been created based on the COMPHEP, PHYTHIA, and JETSET programs. The computation shows the importance of the QCD fake background which was not
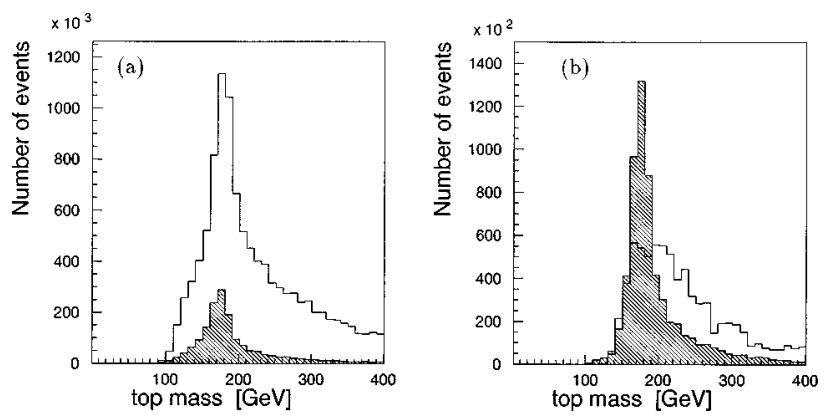

FIG. 7. Distributions for invariant top quark mass before (a) and after (b) cut application at the LHC. The sketched histogram stands for signal. 
taken into account in previous papers. The study of the effects of the initial and final state radiation for $j b b$ process shows that such an approximative method of the simulation of higher jet multiplicity processes has an accuracy of the order of $10 \%$ or less for the rate and gives a significantly softer $p_{T}$ distribution of the radiating jet compared to complete calculations.

It was shown that after optimized cuts were applied the signal from the single top quark can be extracted from the background with a signal to background ratio about 0.4 for the upgraded Tevatron and 1 for the LHC. The single top quark rate in the lepton+jets mode remaining after cuts is expected to be about 120 events at the upgraded Tevatron and about $1.6 \times 10^{5}$ events at the low luminosity LHC operation with $30 \mathrm{fb}^{-1}$ accumulated data and assumptions made above. One can expect that $V t b$ CKM matrix element can be measured at the upgraded Tevatron with an accuracy of about $10 \%$ and hopefully with an accuracy of the order of a few percent at the LHC.

\section{ACKNOWLEDGMENTS}

The authors are grateful to members of the single top quark group of the D0 Collaboration for useful discussions. A.B. is grateful to S. F. Novaes for fruitful discussions, thanks the Instituto de Física Teórica for its kind hospitality, and acknowledges support from Fundação de Amparo à Pesquisa do Estado de São Paulo (FAPESP). E.B. would like to thank H. Anlauf, P. Manakos, T. Ohl, A. Pukhov, V. Savrin, J. Smith, C.-P. Yuan, and B.-L. Young for discussions on different aspects of the calculations used; he wishes to acknowledge the KEK Minami-Tateya (GRACE) Collaboration for kind hospitality during his visit at KEK and his colleagues from the CompHEP group for the interest and support. E.B. and L.D. acknowledge the financial support of the Russian Foundation of Basic Research (Grant No. 96-0219773a), the Russian Ministry of Science and Technologies, and the Sankt-Petersburg Grant Center.
[1] CDF Collaboration, F. Abe et al., Phys. Rev. Lett. 74, 2626 (1995); DØ Collaboration, S. Abachi et al., ibid. 74, 2632 (1995)

[2] Dicus and S. Willenbrock, Phys. Rev. D 34, 155 (1986); C.-P. Yuan, ibid. 41, 42 (1990); G.V. Jikia and S.R. Slabospitsky, Phys. Lett. B 295, 136 (1992); R.K. Ellis and S. Parke, Phys. Rev. D 46, 3785 (1992); G. Bordes and B. van Eijk, Z. Phys. C 57, 81 (1993); D.O. Carlson and C.-P. Yuan, Phys. Lett. B 306, 386 (1993); G. Bordes and B. van Eijk, Nucl. Phys. B435, 23 (1995); S. Cortese and R. Petronzio, Phys. Lett. B 253, 494 (1991); D.O. Carlson, E. Malkawi, and C.-P. Yuan, ibid. 337, 145 (1994); T. Stelzer and S. Willenbrock, ibid. 357, 125 (1995); R. Pittau, ibid. 386, 397 (1996); M. Smith and S. Willenbrock, Phys. Rev. D 54, 6696 (1996); D. Atwood, S. BarShalom, G. Eilam, and A. Soni, ibid. 54, 5412 (1996); D. Atwood, S. Bar-Shalom, and A. Soni, ibid. 57, 2957 (1998); C.S. Li, R.J. Oakes, and J.M. Yang, ibid. 55, 1672 (1997); 55, 5780 (1997); G. Mahlon and S. Parke, ibid. 55, 7249 (1997); A.P. Heinson, A.S. Belyaev, and E.E. Boos, ibid. 56, 3114 (1997); T. Stelzer, Z. Sullivan, and S. Willenbrock, ibid. 56, 5919 (1997); T. Tait and C.-P. Yuan, MSUHEP-71015, 1997, hep-ph/9710372.

[3] R.D. Peccei and X. Zhang, Nucl. Phys. B337, 269 (1990); R.D. Peccei, S. Peris, and X. Zhang, ibid. B349, 305 (1991).

[4] T. Sjöstrand, Comput. Phys. Commun. 82, 74 (1994).

[5] E.E. Boos et al., Report No. SNUTP-94-116, hep-ph/9503280; P. Baikov et al., in Proceedings of the Xth International Workshop on High Energy Physics and Quantum Field Theory, Moscow, 1995, QFTHEP-95, edited by B. Levtchenko and V. Savrin (unpublished), p. 101.

[6] V.A. Ilyin, D.N. Kovalenko, and A.E. Pukhov, Int. J. Mod.
Phys. C 7, 761 (1996); D.N. Kovalenko and A.E. Pukhov, Nucl. Instrum. Methods Phys. Res. A 389, 299 (1997).

[7] S. Kawabata, Comput. Phys. Commun. 41, 127 (1996).

[8] A.P. Heinson, A.S. Belyaev, and E.E. Boos, Phys. Rev. D 56, 3114 (1997).

[9] M. Smith and S. Willenbrock, Phys. Rev. D 54, 6696 (1996); T. Stelzer, Z. Sullivan, and S. Willenbrock, ibid. 56, 5919 (1997).

[10] M. Dittmar and H. Dreiner, Phys. Rev. D 55, 167 (1997).

[11] S. Moretti, Phys. Rev. D 56, 7427 (1997).

[12] N.V. Dokholyan and G.V. Jikia, Phys. Lett. B 336, 251 (1994); E. Boos et al., Z. Phys. C 70, 255 (1996).

[13] E. Laenen, J. Smith, and W.L. van Neereven, Nucl. Phys. B369, 54 (1992); Phys. Lett. B 321, 254 (1994); E.L. Berger and H. Contopanagos, ibid. 361, 115 (1995); Phys. Rev. D 54, 3085 (1996); S. Catani, M. Mangano, P. Nason, and L. Trentadue, Phys. Lett. B 378, 329 (1996); Nucl. Phys. B478, 273 (1996).

[14] A. Belyaev, E. Boos, L. Dudko, and A. Pukhov, "W + 2 Jets Production at Tevatron: Vecbos and CompHEP Comparison,', Report No. D0-NOTE-2784, 1995 (unpublished); hep$\mathrm{ph} / 9511306$.

[15] TeV-2000 Study Group, D. Amidei et al., Report No. FERMILAB-PUB-96-082, 1996.

[16] J. Ohnemus, Phys. Rev. D 44, 3477 (1991).

[17] T. Han and S. Willenbrock, Phys. Lett. B 273, 167 (1991); J. Ohnemus and W.J. Stirling, Phys. Rev. D 47, 2722 (1993); H. Baer, B. Bailey and J. Owens, ibid. 47, 2730 (1993).

[18] D0 Collaboration, S. Abachi et al., Phys. Rev. D 52, 4877 (1995).

[19] C.-P. Yuan, Phys. Rev. D 41, 42 (1990). 